\title{
Recurrent Syncope in a Patient with an Implantable Loop Recorder: What is the Diagnosis?
}

\author{
AMAN CHUGH, M.D., RABEEA ABOUFAKHER, M.D.,* ARTHUR RIBA, M.D.,* \\ and BRADLEY KNIGHT, M.D.
}

From the Division of Cardiology, Department of Internal Medicine, University of Michigan, Ann Arbor, Michigan; and * Department of Internal Medicine, Oakwood Health Care System, Dearborn, Michigan

\section{Case Presentation}

A 76-year-old woman with a history of hypertension and mastectomy for breast carcinoma was referred for evaluation of recurrent syncope. She characterized a typical episode as a sudden loss of consciousness without a prodrome. There was no sensation of fatigue after the events. Her medical regimen consisted of amlodipine and a hydrochlorothiazide/triamterene combination. Results of physical examination were unremarkable. ECG showed sinus rhythm with a left bundle branch block and normal PR and QT intervals. Results of echocardiogram, exercise stress test, 24-hour Holter monitor, and a head-up tilt-table test were normal. She had no syncope, and no arrhythmias were detected while she wore a 30-day external event recorder. Electrophysiologic study including carotid sinus massage was notable only for spontaneous and pacing-induced atrial fibrillation. In view of the nonspecific electrophysiologic study, a loop recorder (Reveal Plus, Medtronic Inc., Minneapolis, MN, USA) was implanted. The loop recorder was

J Cardiovasc Electrophysiol, Vol. 13, pp. 1176-1177, November 2002.

Address for correspondence: Bradley P. Knight, M.D., Division of Cardiology, Department of Internal Medicine, University of Michigan Medical Center, 1500 East Medical Center Drive, B1 F245, Ann Arbor, MI 48109. Fax: 734-936-7026; E-mail: bpk@umich.edu implanted on the right side because the patient had undergone a radical mastectomy and was left with little subcutaneous tissue on the left side.

The patient had an episode of syncope 6 weeks after the loop recorder was implanted. The retrieved electrogram from the patient-activated event is shown in Figure 1. What is the diagnosis of the implantable loop recording?

\section{Commentary}

Interrogation of the loop recorder appears to show atrial fibrillation with a bundle branch block and rapid ventricular response, followed by a nonsustained episode of polymorphic ventricular tachycardia or ventricular fibrillation. However, recurrent syncope due to spontaneously aborted ventricular fibrillation seemed unlikely. To clarify the mechanism of the arrhythmia, the patient was brought to the electrophysiology laboratory, where atrial fibrillation was induced with rapid atrial pacing. AV block was induced with intravenous adenosine, and the implantable loop recorder was activated. The electrogram was retrieved and is shown in Figure 2. The electrogram recorded during AV block reproduced the clinical recording and confirms that the large-amplitude fibrillatory waves are of atrial rather than ventricular origin. The patient was treated with a dual-
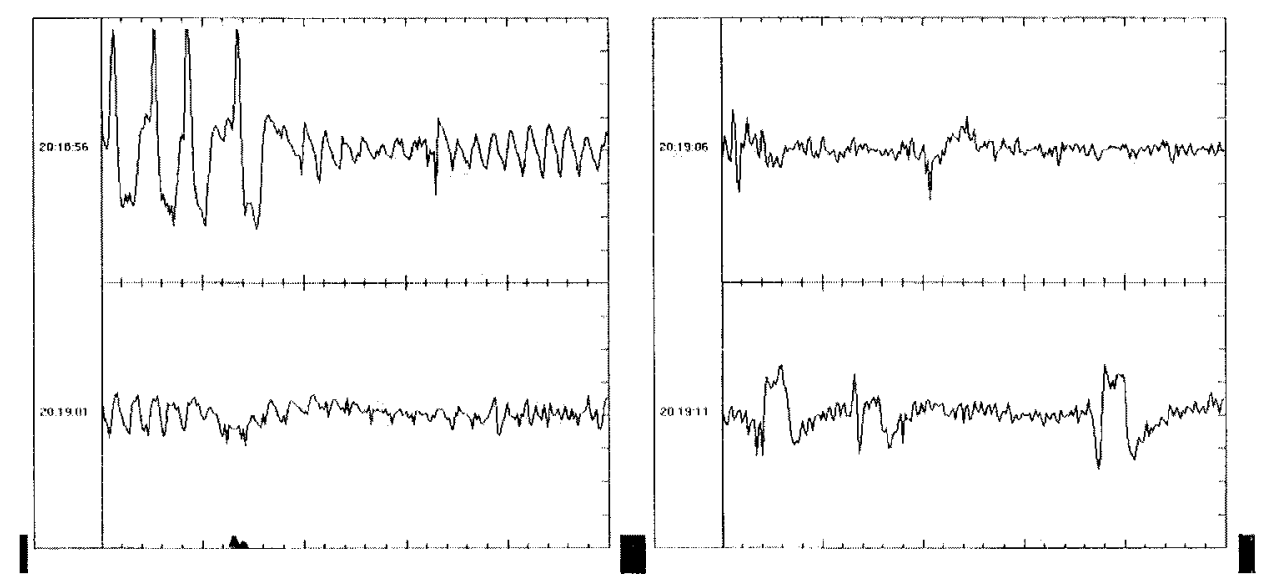

Figure 1. Patient-activated event electrogram retrieved from an implantable loop recorder in a patient experiencing an episode of syncope. The tracing is continuous and was printed at a paper speed of $25 \mathrm{~mm} / \mathrm{sec}$ and gain setting of $8 \times$. 

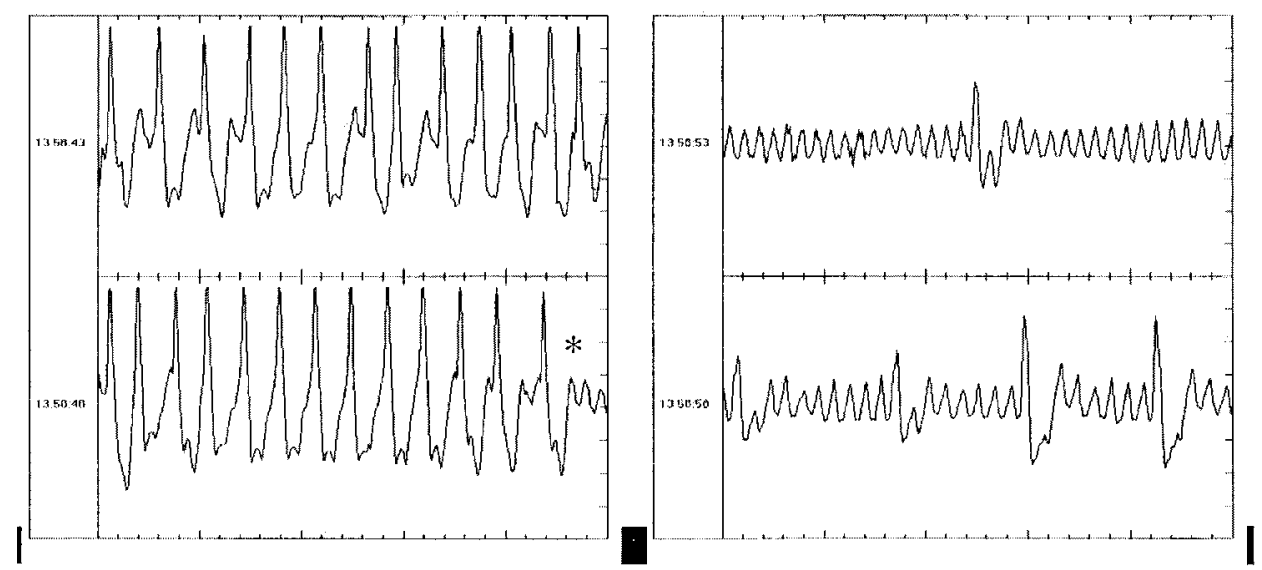

Figure 2. Electrogram retrieved from an implantable loop recorder after induction of atrial fibrillation and administration of intravenous adenosine in the electrophysiologylaboratory. The asterisk indicates the development of AV block. The tracing is continuous and was printed at a paper speed of $25 \mathrm{~mm} / \mathrm{sec}$ and gain setting of $8 \times$ (same as Fig. 1).

chamber pacemaker and amiodarone for intermittent complete heart block triggered by paroxysmal atrial fibrillation.

Atrial fibrillation with high-degree AV block has been shown to mimic ventricular fibrillation during ECG telemetry monitoring. ${ }^{1,2}$ This case demonstrates that atrial fibrillation with complete $\mathrm{AV}$ block can simulate ventricular fibrillation during a recording from an implantable loop recorder. The large amplitude of the atrial fibrillation waves relative to the QRS amplitude may have been due to the right-sided implantation. This case also illustrates the value of an implantable loop recorder in clarifying the diagnosis of recurrent unexplained syncope. ${ }^{3,4}$

\section{References}

1. Katz A, Snir Y, Wagshal A, Cohn G, Ilia R: Pseudoventricular fibrillation. Crit Care Med 2001;29:1835.

2. Ornato JP, Gonzales ER, Garnett R: Atrial fibrillation with high degree atrioventricular block masquerading as ventricular fibrillation masquerading as asystole during cardiac arrest. Crit Care Med 1987;15: 334.

3. Brignole M, Menozzi C, Moya A, et al., on behalf of the International Study on Syncope of Uncertain Etiology (ISSUE) Investigators: Mechanism of syncope in patients with bundle branch block and negative electrophysiological test. Circulation 2001;104:2045.

4. Mieszczanska H, Ibrahim B, Cohen T: Initial clinical experience with implantable loop recorders. J Invas Cardiol 2001;13:802-804. 